\title{
Epidemiology, risk factors, and outcomes of Clostridium difficile infection in kidney transplant recipients
}

\author{
D. Neofytos ${ }^{1}$, K. Kobayashi ${ }^{1,2}$, C.D. Alonso ${ }^{1,3}$, J. Cady-Reh $^{4}$, D. Lepley ${ }^{4}$, M. Harris ${ }^{1}$, N. \\ Desai $^{5}$, E. Kraus ${ }^{1}$, A. Subramanian ${ }^{1}$, S. Treadway ${ }^{1}$, D. Ostrander ${ }^{1}$, C. Thompson ${ }^{6}$, and K. \\ Marr $^{1,7}$ \\ ${ }^{1}$ Department of Medicine, The Johns Hopkins University, School of Medicine, Baltimore, \\ Maryland USA \\ ${ }^{2}$ Department of Medicine, Massachusetts General Hospital, Boston, Massachusetts USA \\ ${ }^{3}$ Department of Medicine, Beth Israel Deaconess Medical Center, Boston, Massachusetts USA \\ ${ }^{4}$ Quality Improvement Department, The Johns Hopkins University, Baltimore, Maryland USA \\ ${ }^{5}$ Department of Surgery, The Johns Hopkins University, Baltimore, Maryland, USA \\ ${ }^{6}$ Biostatistics Center, The Johns Hopkins Bloomberg School of Public Health, Baltimore, \\ Maryland USA \\ ${ }^{7}$ Department of Oncology, The Johns Hopkins University, Baltimore, Maryland, USA
}

\section{Abstract}

Background-We sought to describe the epidemiology and risk factors for Clostridium difficile infection (CDI) among kidney transplant recipients (KTR) between 1 January 2008 and 31 December 2010.

\begin{abstract}
Methods-A single-institution retrospective study was conducted among all adgbult KTR with CDI, defined as a positive test for $C$. difficile, by a cell cytotoxic assay for $C$. difficile toxin A or $\mathrm{B}$ or polymerase chain reaction test for toxigenic $C$. difficile.
\end{abstract}

\begin{abstract}
Results-Among 603 kidney transplants performed between 1 January 2008 and 31 December 2010, 37 (6.1\%) patients developed CDI: 12 (of 128; 9.4\%) high-risk (blood group incompatible and/or anti-human leukocyte antigen donor-specific antibodies) vs. 25 (of $475 ; 5.3 \%, P=0.08$ ) standard-risk patients. The overall rate of CDI increased from $3.7 \%$ in 2008 to $9.4 \%$ in 2010 ( $P=$ 0.05). The median time to CDI diagnosis was 9 days, with 27 (73.0\%) patients developing CDI within the first 30 days after their transplant, and 14 (51.8\%) developing CDI within 7 days. A case-control analysis of $37 \mathrm{CDI}$ cases and 74 matched controls demonstrated the following predictors for CDI among KTR: vancomycin-resistant Enterococcus colonization before transplant (odds ratio [OR]: 3.6, $P=0.03$ ), receipt of an organ from a Centers for Disease Control high-risk donor (OR: 5.9, $P=0.006$ ), and administration of high-risk antibiotics within 30 days post transplant (OR: 6.6, $P=0.001)$.
\end{abstract}

\footnotetext{
Correspondence to: Dionissios Neofytos, MD, MPH, Transplant and Oncology Infectious Disease Program, The Johns Hopkins School of Medicine, Division of Infectious Diseases, 1830 E Monument Street, Suite 421, Baltimore, MD 21205 USA, Tel: 410502 9521, Fax: 410614 0714, dneofyt1@jhmi.edu.

Conflicts of Interest: D.N. has received research grants from Pfizer. K.A.M. has received grant support from Astellas, Merck and Pfizer and have served on advisory boards/or as consultant for Astellas, Merck, Optimer, and Pfizer. All other authors: No conflicts of interest.
} 
Conclusions-CDI remains a common early complication in KTR, with rates steadily increasing during the study period. Host and transplant-related factors and exposure to antibiotics appeared to significantly impact the risk for CDI among KTR.

\section{Keywords}

Clostridium difficile; kidney transplant

Nosocomial Clostridium difficile infection (CDI) is a significant problem that has worsened with the emergence of the NAP1/BI/027 (binary toxin) strain (1-7). Advanced age, antibiotic use, and immunosuppression (both acquired and inherited) are established risk factors that make hospitalized patients susceptible to CDI (1-7). Solid organ transplant (SOT) patients are a high-risk patient population for CDI because of their immunosuppressive regimens and increased frequency and duration of hospitalizations. The incidence of CDI among SOT recipients has ranged between $1.74 \%$ and $7.9 \%(8-10)$. Recent data on the incidence, timing, and risk factors for CDI among kidney transplant recipients (KTR) are lacking. This information is pertinent to the management and outcome optimization of these high-risk patients. We sought to review the epidemiology, timing, and outcomes of CDI and assess risk factors of CDI among KTR between 1 January 2008 and 31 December 2010.

\section{Methods}

\section{Study design and patient population}

The study was approved by the Institutional Review Board of the Johns Hopkins Hospital and was performed as part of a Quality Assessment and Performance Improvement project. In this retrospective study, all adult (age $\geq 18$ years) patients that received a kidney transplant between 1 January 2008 and 31 December 2010 were identified using the database system of the Comprehensive Transplant Center (of the Johns Hopkins Hospital. Transplant recipients with a diagnosis of CDI, who were admitted to the same ward exclusively caring for kidney (and liver) transplant recipients during the study period, were identified by review of the databases of the Hospital Epidemiology Infection Control and the Comprehensive Transplant Center. Patients with CDI from day -7 of transplant to 6 months after transplantation were included. To specifically study risk factors for CDI among KTR, patients with CDI during the study period were identified as cases and were matched 1:2 to controls (no evidence of CDI) by day of transplant ( \pm 3 days).

\section{Immunosuppression and prophylactic regimen}

All KTR at our institution receive induction immunosuppression, as per type-dependent protocol. Maintenance immunosuppression includes prednisone, tacrolimus, and mycophenolate mofetil (MMF). On occasion, based upon clinical indications, sirolimus rather than tacrolimus is used. Human leukocyte antibody (HLA) incompatible (positive crossmatch) KTR receive alternate-day plasmapheresis and $100 \mathrm{mg} / \mathrm{kg}$ of intravenous hyperimmune globulin (Cytogam, Medimmune, Inc., Gaithersburg, Maryland USA) before transplantation to remove donor-specific anti-HLA antibody, and quadruple-drug immunosuppression (daclizumab, tacrolimus or sirolimus, MMF, and steroids) as previously described $(11,12)$. Patients with persistent donor-specific antibody may additionally receive anti-CD20 antibody therapy or/and splenectomy. Blood group incompatible live donor KTR receive an anti-interleukin-2r antibody therapy.

Routine preoperative antibiotics, including cefazolin or vancomycin (for penicillin-allergic patients), are administered to all patients. Pneumocystis prophylaxis is initiated immediately 
post transplant with trimethoprim-sulfamethoxazole (TMP/SMX) and continued for 6 months; atovaquone or dapsone may be used in patients with a history of allergic reaction to sulfa drugs or other reasons, as deemed suitable by the treating physicians. Prophylaxis against cytomegalovirus (CMV) with ganciclovir or valganciclovir is initiated post transplant and continued for 3 and 6 months for CMV-positive recipients and CMV-positive donor/CMV-negative recipients, respectively. Antifungal prophylaxis consists of clotrimazole troches for 30 days post transplant. All patients receive gastric prophylaxis with either a proton pump inhibitor or a histamine-2 blocker.

\section{Clinical variables}

The following host variables were collected for all patients: demographics, comorbidities (diabetes mellitus, coronary artery disease, liver disease), type and number of prior organ transplants, underlying disease leading to transplant, surveillance cultures for vancomycinresistant Enterococcus (VRE) upon admission, and hospital and intensive care unit (ICU) days within the first 30 days post transplant. History and years of renal replacement therapy (hemodialysis or/and peritoneal dialysis) before kidney transplant were recorded. The following laboratory variables were collected: serum creatinine on days 1 and 7 post transplant, peak WBC count, and nadir absolute lymphocyte count within the first 30 days post transplant. Administration of high-risk antibiotics during the first 30 days post transplant and the number of days of antibiotic administration were recorded $(13,14)$. Transplant-related variables included the following: day of transplant, induction and maintenance immunosuppression regimens, transplant risk (standard vs. high-risk, defined as blood type or/and HLA incompatible donor-recipients), and donor status (deceased vs. live donor, related vs. unrelated live donor, Center of Disease Control [CDC] high-risk vs. not), and donor and recipient CMV serology status (15).

CDI-related variables included the diagnostic test used, presence of fever (temperature $>38.3^{\circ} \mathrm{C}$ ), toxic megacolon, transfer to the ICU, treatments administered, and recurrent disease. Radiologic reports were evaluated for the presence of colonic distention, colonic wall thickening, pericolic fat stranding, and ascites. Physician notes and the electronic medical record were evaluated for the results of colonoscopies or flexible sigmoidoscopies.

\section{Definitions}

CDI was defined as a positive stool sample for $C$. difficile. Testing for $C$. difficile included a cell cytotoxic assay for C. difficile toxin A or B (until 1 October 2009) or a polymerase chain reaction (PCR) targeting the toxigenic C. difficile toxin $\mathrm{B}$ gene, thereafter. Recurrent CDI was defined as a new CDI episode at least 14 days after the completion of treatment for the primary CDI event (16). Severe CDI was defined by the presence of toxic megacolon, colitis requiring colectomy, or admission/transfer to the ICU because of CDI. High-risk antibiotics were defined as anti-pseudomonal penicillins, third- and fourth-generation cephalosporins, carbapenems, fluoroquinolones, and clindamycin $(13,14)$. Blood group incompatible and/or positive crossmatch KTR were defined as high-risk transplants.

\section{Statistical analysis}

Among the overall population of KTR between 2008 and 2010, rates of infection were calculated by dividing the number of patients who developed CDI over the total number of transplants performed in the cohort year. A test for linear trend was used to compare differences in rates of infection by year of transplant. Two-sided $P$-values of $₫ 0.05$ were considered significant. Time to CDI after transplant was calculated using the first posttransplant episode of CDI. Using the case-control study population sample, we sought to identify risk factors for CDI among KTR. Variables that had a $\mathrm{p}$ value $<0.10$ in the univariate analyses were introduced into a stepwise multivariate logistic regression model to 
assess risk factors. Odds ratios (ORs) and 95\% confidence intervals (CIs) are presented for all logistic regression analysis results. Statistical analyses were performed using Stata software v 11.12010 (StataCorp, College Station, Texas USA).

\section{Results}

\section{Rates and timing of CDI}

A total of 603 kidney transplants (475 standard and 128 high-risk) were performed at our institution between 1 January 2008 and 31 December 2010. The overall rate of CDI among all KTR was $6.1 \%$ (37 of 603). A trend was seen for more high-risk KTR to develop CDI ( 12 of $128 ; 9.4 \%$ ) compared with standard KTR ( 25 of $475 ; 5.3 \%, P=0.08)$. The rate of CDI increased from 3.7\% (6 of 161) in 2008 to $9.4 \%$ (19 of 201, $P=0.05$ ) in 2010 among all KTR. This increase was mainly driven by higher yearly rates of CDI among standard-risk KTR, from $2.4 \%$ ( 3 of 125 ) in 2008 to $10.6 \%$ (16 of 151 ) in 2010 ( $P=0.002$; Fig. 1). The rates of CDI among high-risk KTR remained high, but relatively stable, during the study period.

The median time to CDI diagnosis was 9 days post transplant (range: $-6,338$ ) for all KTR. Notably, 27 (73.0\%) patients developed CDI within the first 30 days after their transplant, and $14(51.8 \%)$ of them within the first 7 days post transplant (Fig. 2). Recipients who received a transplant from a related donor appeared to develop CDI later (median: 170.9 days, range: 3,338 ) compared with unrelated-donor KTR (median: 9 days, range: $-6,123, P$ $<0.001$ ). No significant differences were seen in timing to CDI diagnosis based on the kidney transplant risk status or other transplant related variables (alive vs. deceased, CDC high-risk donor vs. not; data not shown).

\section{Case-control study}

Of the $603 \mathrm{KTR}, 37 \mathrm{CDI}$ cases and 74 matched controls were included in the case-control analysis. Demographics and clinical characteristics of the patients are described in Table 1. More cases were colonized with VRE ( $43.2 \%$ vs. $17.6 \%, P=0.004)$ and had longer hospital (mean: 20.6 vs. 13.8 days, $P=<0.001$ ) and ICU stay (mean: 3.1 vs. 1.5 days, $P=0.005$ ).

Transplant variables differed between cases and controls, with more cases having received a transplant from a deceased $(73.0 \%$ vs. $52.7 \%, P=0.04)$ or CDC high-risk donor $(40.5 \%$ vs. $14.9 \%, P=0.003)$ and anti-thymocyte globulin for induction $(97.3 \%$ vs. $83.8 \%, P=0.02)$. A greater proportion of case patients had received intravenously administered vancomycin ( $75.7 \%$ vs. $18.9 \%, P<0.001)$ and $\geq 1$ high-risk antibiotic agents $(83.8 \%$ vs. $44.6 \%, P=0.007)$ compared with controls. One case and 2 controls had a diagnosis of CDI 2 years and 3 and 5 months, respectively, before their transplant.

Independent variables studied to identify risk factors for CDI in univariate analyses are presented in Table 2 . When variables with $P \unlhd 0.10$ were introduced in a stepwise multivariate logistic regression model, the major predictors for CDI among KTR were the following: prior VRE colonization (OR: 3.6, 95\% CI: 1.1, 11.3, $P=0.03$ ), CDC high-risk donor (OR: 5.9, 95\% CI: 1.7, 20.8, $P=0.006$ ), and administration of high-risk antibiotics (OR: 6.6, 95\% CI: 2.1, 21.0, $P=0.001$ ).

\section{Clinical presentation of CDI and treatment}

Three (of 37, 8.1\%) patients had a fever and 2 required admission to the ICU attributed to CDI. One of these 2 patients had evidence of toxic megacolon. None of the patients with $\mathrm{CDI}$ in this series had a colonoscopy. Owing to the retrospective nature of the study, we were not able to collect data on the presence and severity of diarrhea on these patients. A white blood cell (WBC) count $>20,000$ cells $/ \mathrm{mm}^{3}$ was observed in $9(24.3 \%)$ patients. Most 
patients with CDI were lymphopenic with a mean absolute lymphocyte count of 96.6 cells/ $\mathrm{mm}^{3}$. The majority of patients $(32,86.5 \%)$ were treated with a single agent: 27 (of 32, $84.4 \%)$ with oral vancomycin and $5(16.6 \%)$ with metronidazole (either intravenously or orally). Among 32 patients treated with oral vancomycin, 20 and 12 patients were administered $250 \mathrm{mg}$ and $125 \mathrm{mg} 4$ times daily, respectively. Five (13.5\%) patients were treated with combination therapy including orally administered vancomycin and metronidazole (either orally or intravenously). The median duration of treatment was 15 days (range: 10-42). Only one patient received treatment with intravenous hyperimmune globulin.

Among 37 patients with CDI, $4(10.8 \%)$ were diagnosed with another CDI episode at a median time of 235.5 days (range: 27-307). All KTR with CDI were alive by 6 months after their CDI diagnosis.

\section{Discussion}

Our observations suggest that CDI remains a common early complication in a contemporary cohort of KTR, with rates steadily increasing during the study period. The higher rates of CDI observed among KTR and the overall hospital patient population (personal communication with Hospital Epidemiology and Infection Control) during the study period may, in part, be related to the more sensitive test (PCR) used for the diagnosis of CDI that was implemented after October 2009. PCR testing may lead to more false-positive diagnoses of CDI, as a positive stool test for $C$. difficile by PCR may imply CDI or simply colonization with $C$. difficile, depending on the setting. In fact, the majority of CDI cases in KTR were diagnosed within 30 days (half of them within a week) after their transplant. It is likely that some of them (i) might have acquired $C$. difficile in the past, from multiple exposures to the healthcare setting for renal replacement therapies or other complications of their underlying disease before their admission, or (ii) they did not have CDI, but their diarrhea was rather a result of other causes, including medications (e.g., MMF, antibioticrelated diarrhea) or other infections. Colonization with $C$. difficile has been described in healthy outpatients, hospitalized patients, and patients in long-term care facilities at rates up to $1-2 \%, 10-30 \%$, and $10-30 \%$, respectively (17-23). Hence, we hypothesize that a subset of patients may already be colonized with $C$. difficile coming in for their transplant. The impact of $C$. difficile asymptomatic colonization on the risk for active disease should be further studied in prospective studies.

High-risk KTR, defined as ABO or/and HLA incompatible, appeared to have the highest rates of CDI, averaging $11.5 \%$ during the study period. This finding may, in part, be related to the higher degree of immunosuppression required for these patients. Recent data suggest that B-cell immunity, particularly immunogloblulin-G response to toxin $A$, is crucial to contain CDI $(24,25)$. In addition to their profound T-cell suppression, some of these highrisk kidney recipients received B-cell suppressing agents, including rituximab, daclizumab, or/and plasmapheresis. Moreover, these patients may require more frequent and longer hospitalizations for post-transplant complications, such as rejection, increasing their exposure to the healthcare environment and risks for acquiring $C$. difficile. Notably, the incidence of CDI among high-risk KTR did not change during the study period, perhaps, in part, as a result of healthcare personnel being more careful when caring for sicker, more immunosuppressed patients, and applying infection control practices in a stricter way.

As previously reported, administration of high-risk antibacterial agents was the most significant predictor of CDI in KTR (26-28). Colonization with VRE before a kidney transplant was another significant predictor for CDI in this cohort, as previously described in hematopoietic stem cell transplant recipients (28). Notably, in a review of 18 case-control 
studies, similar risk factors, including antimicrobial exposure, number of antimicrobials, days of antimicrobial use, and specific agents (third-generation cephalosporins, clindamycin, and imipenem) were identified for both VRE infection/colonization and CDI (27). It is likely that VRE colonization may, in part, represent a surrogate marker of underlying disease severity and exposure to healthcare system, both leading to higher risks for CDI. Receipt of an organ from a CDC high-risk donor was a strong predictor for CDI. This may, in part, be attributed to the status of patients who are more willing to accept an organ from a high-risk $\mathrm{CDC}$ donor, including patients at high risk of death or with poor quality of life on dialysis $(28,29)$.

KTR are administered medicines with potential bone marrow toxicity (e.g., MMF, TMP/ SMX, or ganciclovir) that may prevent them from mounting a high WBC count. In fact, only a minority of patients $(9 ; 24.3 \%)$ had a WBC $\geq 20,000$ cells $/ \mathrm{mm}^{3}$. Another potential explanation of the lower WBC counts observed in this series may be the fact that a number of patients were colonized with $C$. difficile, rather than having disease. In fact, only 1 patient had severe $\mathrm{CDI}$, presenting with toxic megacolon and requiring colectomy and transfer to the ICU in this series. In total, $10.8 \%$ of KTR with CDI had a CDI relapse, consistent with relapse rates in general hospitalized population and data from hematopoietic stem cell transplant recipients (30).

This was a retrospective 3-year observational study with inherent limitations, including the small sample of patients and limited data availability. The definition of CDI was based on a positive stool test rather than established guidelines (31). Owing to the retrospective nature of this study, we were not able to collect more specific information about the consistency and frequency of stools to accurately define diarrhea. Finally, disparities in patient follow-up (e.g., closer monitoring of high-risk kidney transplants) and thus more frequent CDI testing may explain some of the differences in CDI rates among kidney transplant types.

Overall, we report a $6.1 \%$ incidence of CDI among KTR, with ABO or/and HLA incompatible kidney transplants demonstrating the highest rates of CDI. The early diagnosis of CDI in the majority of KTR raises concerns about $C$. difficile colonization on admission. The impact of the latter on the development of active disease after exposure to conditioning agents and preoperative antibacterial therapy early post transplant remains to be defined. Prospective studies to identify colonized patients pre-transplant and associated risks for active disease may improve our understanding of the natural history of this infection and enable the development of preventive strategies in the future.

\section{Acknowledgments}

Thanks: The authors would like to thank Ramon Konewko, Brigitte Sullivan, Vidhya Gunaseelan, and the Quality Improvement Department for their support.

Support: The study was in part supported by a grant from the NIH: K24 AI085118. Statistical analysis was, in part, supported by a National Center for Research Resources and the National Center for Advancing Translational Sciences (NCATS) of the National Institutes of Health Grant (101872).

\section{References}

1. Kyne L, Hamel MB, Polavaram R, Kelly CP. Health care costs and mortality associated with nosocomial diarrhea due to Clostridium difficile. Clin Infect Dis. 2002; 34:346-353. [PubMed: 11774082]

2. Dubberke ER, Wertheimer AI. Review of current literature on the economic burden of Clostridium difficile infection. Infect Control Hosp Epidemiol. 2009; 30:57-66. [PubMed: 19049438] 
3. Bobak D, Arfons LM, Creger RJ, Lazarus HM. Clostridium difficile-associated disease in human stem cell transplant recipients: coming epidemic or false alarm? Bone Marrow Transplant. 2008; 42:705-713. [PubMed: 18836490]

4. McDonald LC, Killgore GE, Thompson A, et al. An epidemic, toxin gene-variant strain of Clostridium difficile. N Engl J Med. 2005; 353:2433-2441. [PubMed: 16322603]

5. Freeman J, Bauer MP, Baines SD, et al. The changing epidemiology of Clostridium difficile infections. Clin Microbiol Rev. 2010; 23:529-549. [PubMed: 20610822]

6. Loo VG, Poirier L, Miller MA, et al. A predominantly clonal multi-institutional outbreak of Clostridium difficile-associated diarrhea with high morbidity and mortality. N Engl J Med. 2005; 353:2442-2449. [PubMed: 16322602]

7. Thibault A, Miller MA, Gaese C. Risk factors for the development of Clostridium difficileassociated diarrhea during a hospital outbreak. Infect Control Hosp Epidemiol. 1991; 12:345-348. [PubMed: 2071877]

8. Keven K, Basu A, Re L, et al. Clostridium difficile colitis in patients after kidney and pancreaskidney transplantation. Transpl Infect Dis. 2004; 6 (1):10-4. [PubMed: 15225221]

9. Stelzmueller I, Goegele H, Biebl M, et al. Clostridium difficile colitis in solid organ transplantation--a single-center experience. Dig Dis Sci. 2007; 52 (11):3231-3236. [PubMed: 17406820]

10. Albright JB, Bonatti H, Mendez J, et al. Early and late onset Clostridium difficile-associated colitis following liver transplantation. Transpl Int. 2007; 20 (10):856-66. [PubMed: 17854444]

11. Montgomery RA, Locke JE, King KE, et al. ABO incompatible renal transplantation: a paradigm ready for broad implementation. Transplantation. 2009; 87 (8):1246-55. [PubMed: 19384174]

12. Montgomery RA, Lonze BE, King KE, et al. Desensitization in HLA-incompatible kidney recipients and survival. N Engl J Med. 2011; 365 (4):318-26. [PubMed: 21793744]

13. Bartlett JG. Clostridium difficile: progress and challenges. Ann N Y Acad Sci. 2010; 1213:62-69. [PubMed: 21175676]

14. Bouza E, Burillo A, Munoz P. Antimicrobial therapy of Clostridium difficile-associated diarrhea. Med Clin North Am. 2006; 90:1141-1163. [PubMed: 17116441]

15. Rogers, MF.; Lawton, KE.; Moseley, RR.; Jones, WK. Guidelines for Preventing Transmission of Human Immunodeficiency Virus Through Transplantation of Human Tissue and Organs. 1994. Cited 2008 September 12th; Available from: http://www.cdc.gov/MMWR/preview/mmwrhtml/ 00031670.htm

16. Kelly CP. A 76-year-old man with recurrent Clostridium difficile-associated diarrhea: review of $C$. difficile infection. JAMA. 2009; 301:954-962. [PubMed: 19190304]

17. Bender BS, Bennett R, Laughon BE, et al. Is Clostridium difficile endemic in chronic-care facilities? Lancet. 1986; 2 (8497):11-13. [PubMed: 2873315]

18. McFarland LV, Mulligan ME, Kwok RY, Stamm WE. Nosocomial acquisition of Clostridium difficile infection. N Engl J Med. 1989; 320 (4):204-210. [PubMed: 2911306]

19. Johnson S, Clabots CR, Linn FV, Olson MM, Peterson LR, Gerding DN. Nosocomial Clostridium difficile colonisation and disease. Lancet. 1990; 336 (8707):97-100. [PubMed: 1975332]

20. Simor AE, Yake SL, Tsimidis K. Infection due to Clostridium difficile among elderly residents of a long-term-care facility. Clin Infect Dis. 1993; 17 (4):672-678. [PubMed: 7903557]

21. Simor AE, Bradley SF, Strausbaugh LJ, Crossley K, Nicolle LE. SHEA Long-Term-Care Committee. Clostridium difficile in long-term-care facilities for the elderly. Infect Control Hosp Epidemiol. 2002; 23 (11):696-703. [PubMed: 12452300]

22. Muto CA. Asymptomatic Clostridium difficile colonization: Is this the tip of another iceberg? Clin Infect Dis. 2007; 45 (8):999-1000. [PubMed: 17879914]

23. Ryan J, Murphy C, Twomey C, et al. Asymptomatic carriage of Clostridium difficile in an Irish continuing care institution for the elderly: prevalence and characteristics. Ir J Med Sci. 2010; 179 (2):245-250. [PubMed: 19495833]

24. Kyne L, Warny M, Qamar A, Kelly CP. Asymptomatic carriage of Clostridium difficile and serum levels of IgG antibody against toxin A. N Engl J Med. 2000; 342:390-397. [PubMed: 10666429] 
25. Kyne L, Warny M, Qamar A, Kelly CP. Association between antibody response to toxin A and protection against recurrent Clostridium difficile diarrhoea. Lancet. 2001; 357:189-193. [PubMed: 11213096]

26. Gerding DN, Olson MM, Peterson LR, et al. Clostridium difficile-associated diarrhea and colitis in adults. A prospective case-controlled epidemiologic study. Arch Intern Med. 1986; 146:95-100. [PubMed: 3942469]

27. Gerding DN. Is there a relationship between vancomycin-resistant enterococcal infection and Clostridium difficile infection? Clin Infect Dis. 1997; S2:S206-210. [PubMed: 9310680]

28. Alonso CD, Treadway SB, Hanna DB, et al. Epidemiology and outcomes of Clostridium difficile infections in hematopoietic stem cell transplant recipients. Clin Infect Dis. 2012; 54 (8):10531063. [PubMed: 22412059]

29. Gordon EJ, Reddy E, Ladner DP, et al. Kidney transplant candidates' understanding of increased risk donor kidneys: a qualitative study. Clin Transplant. 2012; 26 (2):359-368. [PubMed: 21995601]

30. Ros RL, Kucirka LM, Govindan P, et al. Patient attitudes toward CDC high infectious risk donor kidney transplantation: inferences from focus groups. Clin Transplant. 2012; 26 (2):247-253. [PubMed: 21554396]

31. Louie TJ, Miller MA, Mullane KM, et al. Fidaxomicin versus vancomycin for Clostridium difficile infection. N Engl J Med. 2011; 364:422-431. [PubMed: 21288078]

32. Cohen SH, Gerding DN, Johnson S, et al. Clinical practice guidelines for Clostridium difficile infection in adults: 2010 update by the Society for Healthcare Epidemiology of America (SHEA) and the Infectious Diseases Society of America (IDSA). Infect Control Hosp Epidemiol. 2010; 31 (5):431-455. [PubMed: 20307191] 


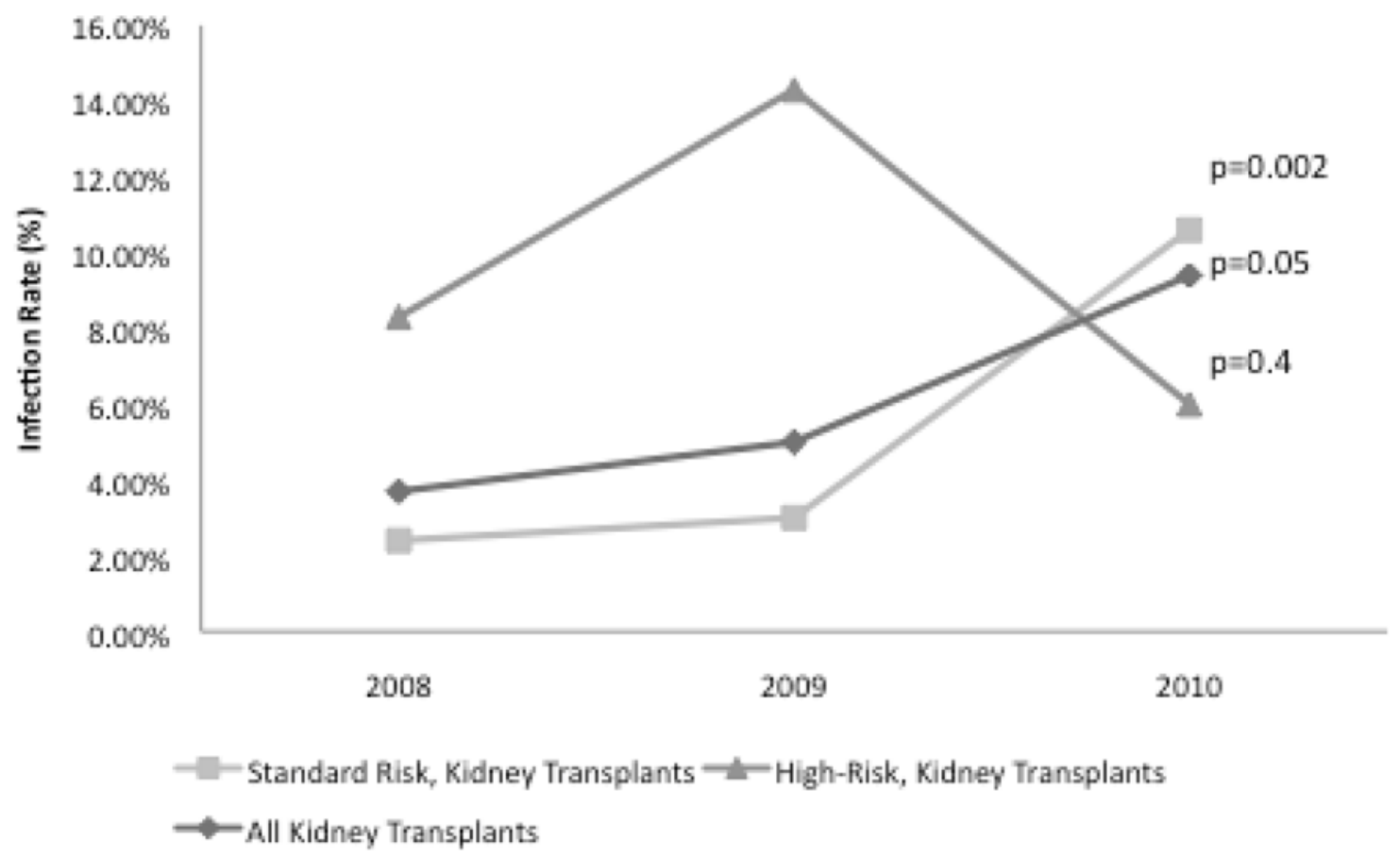

Fig. 1.

Rates of Clostridium difficile infection (CDI) -calculated by number of CDI cases/number of kidney transplant per year, among all (standard and high-risk) kidney transplant recipients between 1 January 2008 and 31 December 2010. $P$-values were calculated with the chi square test for 1$)$ all kidney $(P=0.05), 2)$ standard risk kidney $(P=0.002)$, and 3$)$ high-risk kidney transplant recipients $(P=0.4)$ with CDI between 2008 and 2010. 


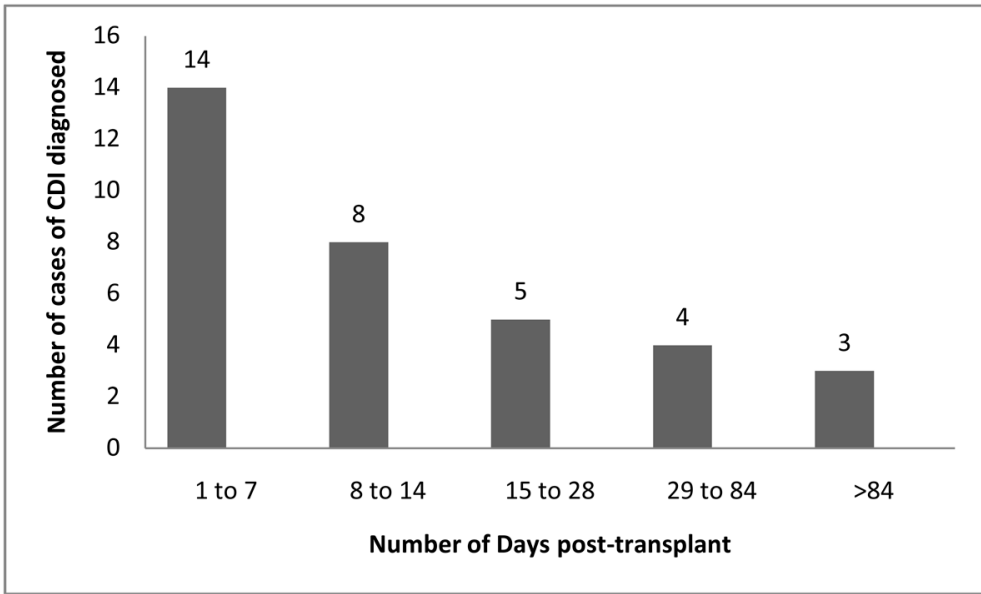

Fig. 2 .

Time to diagnosis of Clostridium difficile infection (CDI) for all kidney transplant recipients between 1 January 2008 and 31 December 2010. 
Table 1

Characteristics of kidney transplant recipients with (cases) and without (controls) Clostridium difficile infection(CDI)

\begin{tabular}{|c|c|c|c|}
\hline Variables & $\begin{array}{c}\text { Cases } \\
\mathbf{N}=\mathbf{3 7}(\%)\end{array}$ & $\begin{array}{l}\text { Controls } \\
\mathrm{N}=74(\%)\end{array}$ & $P$ value \\
\hline \multicolumn{4}{|l|}{ Host variables } \\
\hline \multicolumn{4}{|l|}{ Demographics } \\
\hline Gender, Female & $12(32.4)$ & $36(48.6)$ & 0.8 \\
\hline Ethnicity, Caucasian & $14(37.8)$ & $40(54.0)$ & 0.1 \\
\hline Age (mean; range) & $50.9(18-75)$ & $49.1(18-77)$ & 0.1 \\
\hline \multicolumn{4}{|l|}{ Comorbidities } \\
\hline Diabetes mellitus & $28(75.7)$ & $58(78.4)$ & 0.7 \\
\hline Cardiovascular disease & $6(16.2)$ & $19(25.8)$ & 0.2 \\
\hline Liver disease & $7(18.9)$ & $12(16.2)$ & 0.7 \\
\hline VRE colonization on admission & $16(43.2)$ & $13(17.6)$ & 0.005 \\
\hline Prior transplant, Kidney & $11(29.7)$ & $11(14.9)$ & 0.07 \\
\hline Renal replacement therapy prior to transplant 1 & $31(88.6)$ & $49(73.1)$ & 0.08 \\
\hline Years of renal replacement therapy (mean, range) & $4.8(0-32)$ & $2.9(0-12)$ & 0.07 \\
\hline \multicolumn{4}{|l|}{ Transplant-related variables } \\
\hline High-risk $^{2}$ & $12(32.4)$ & $17(23.0)$ & 0.3 \\
\hline Donor, Deceased & $27(73.0)$ & $39(52.7)$ & 0.04 \\
\hline Donor, Related & $9(24.3)$ & $5(6.8)$ & 0.1 \\
\hline Donor, CDC high-risk ${ }^{2}$ & $15(40.5)$ & $11(14.9)$ & 0.004 \\
\hline \multicolumn{4}{|l|}{ Immunosuppression, Induction } \\
\hline Thymoglobulin & $36(97.3)$ & $62(83.8)$ & 0.02 \\
\hline Monoclonal antibodies ${ }^{3}$ & $10(27.0)$ & $20(27.0)$ & 1.0 \\
\hline \multicolumn{4}{|l|}{ Immunosuppression, Maintenance } \\
\hline Corticosteroids & $37(100)$ & $72(97.3)$ & 0.3 \\
\hline Tacrolimus & 35 (94.6) & $73(98.6)$ & 0.2 \\
\hline Mycophenolate mofetil & $37(100)$ & $72(97.3)$ & 0.3 \\
\hline CMV status & & & 0.5 \\
\hline Recipient + & $25(67.6)$ & $48(64.9)$ & \\
\hline Donor +/Recipient - & $6(16.2)$ & $8(10.8)$ & \\
\hline \multicolumn{4}{|l|}{ Other variables } \\
\hline Number of inpatient days ${ }^{*}, 4$, (mean; range) & $20.6(7-30)$ & $13.8(2-30)$ & $<0.001$ \\
\hline Number of ICU days ${ }^{*}, 4$, (mean; range) & $3.1(0-15)$ & $1.5(0-7)$ & 0.0009 \\
\hline \multicolumn{4}{|l|}{ Laboratory findings } \\
\hline Diagnostic test for CDI, PCR & $31(83.8)$ & NA & \\
\hline Creatinine $(\mathrm{mg} / \mathrm{dL})$, day $1,(\text { mean; range })^{5}$ & $8.7(2.3-23.5)$ & $7.4(1-16.8)$ & 0.15 \\
\hline Creatinine $(\mathrm{mg} / \mathrm{dL})$, day $7,(\text { mean; range })^{6}$ & $3.9(0.2-15.1)$ & $2.8(0.2-12.8)$ & 0.08 \\
\hline
\end{tabular}




\begin{tabular}{|c|c|c|c|}
\hline Variables & $\begin{array}{c}\text { Cases } \\
\mathrm{N}=37(\%)\end{array}$ & $\begin{array}{l}\text { Controls } \\
\mathrm{N}=74(\%)\end{array}$ & $P$ value \\
\hline Peak WBC (cells $\left./ \mathrm{mm}^{3}\right)$, (mean; range) & $18696(7530-77500)$ & $15520(6970-34030)$ & 0.06 \\
\hline Nadir ALC (cells $\left./ \mathrm{mm}^{3}\right),(\text { mean; range })^{7}$ & $96.6(0-480)$ & $285.6(0-2220)$ & 0.02 \\
\hline \multicolumn{4}{|l|}{ High risk antibiotics 4,8} \\
\hline Antipseudomonal penicillins & $13(35.1)$ & $19(25.7)$ & 0.3 \\
\hline Cephalosporins 3rd-4thgeneration & $6(16.2)$ & $8(10.8)$ & 0.4 \\
\hline Carbapenems & $6(16.2)$ & $5(6.7)$ & 0.11 \\
\hline Vancomycin (intravenous) & $28(75.7)$ & $14(18.9)$ & $<0.001$ \\
\hline Fluoroquinolones & $15(40.5)$ & $16(21.6)$ & 0.04 \\
\hline Clindamycin & $3(8.1)$ & $5(6.7)$ & 0.8 \\
\hline Number of high-risk antibiotics ${ }^{4}, \geq 1$ agents & $31(83.8)$ & $33(44.6)$ & 0.007 \\
\hline Days to CDI diagnosis post transplant (median; IQR) & $9(25.2)$ & NA & \\
\hline Mortality at 6 months post CDI & 0 & $6(8.1)$ & 0.03 \\
\hline
\end{tabular}

* Based on the early occurrence of CDI, number of inpatient and ICU days were not included in the multivariate logistic regression analysis.

${ }^{1}$ Data on renal replacement therapy prior to transplant were available for 35 and 67 cases and controls, respectively.

2 High-risk kidney transplants: blood type or/and HLA incompatible donor-recipient transplants. High-risk CDC were defined based on $(28,29)$.

3 Monoclonal antibodies included daclizumab, basiliximab, rituximab, and eculizumab. Patients could have received more than of these agents.

4 From day of transplant (day=1) to 30 days post transplant.

5 Data on creatinine on day 1 were available for 36 and 74 cases and controls, respectively.

6 Data on creatinine on day 7 were available for 35 and 71 cases and controls, respectively.

7 Data on ALC nadir were available for 36 and 73 cases and controls, respectively.

${ }^{8}$ High-risk antibiotics included anti-pseudomonal penicillins, third-and fourth-generation cephalosporins, carbapenems, fluoroquinolones, and clindamycin $(13,14)$.

VRE, vancomycin-resistant Enterococcus; ICU, intensive care unit; CDI, Clostridium difficile infection; CMV, cytomegalovirus; NA, not applicable; WBC, white blood cell count; ALC, absolute lymphocyte count; HLA, human leukocyte antigen; CDC, Centers for Disease Control and Prevention. 
